\title{
Vasculogenic mimicry in head and neck tumors: a narrative review
}

\author{
Yuan Yue^, Yunfan Lou, Xiong Liu, Xiaohong Peng \\ Department of Otolaryngology, Head and Neck Surgery, Nanfang Hospital, Southern Medical University, Guangzhou, China \\ Contributions: (I) Conception and design: X Peng; (II) Administrative support: X Liu; (III) Provision of study materials or patients: Y Lou; (IV) \\ Collection and assembly of data: Y Yue; (V) Data analysis and interpretation: Y Yue; (VI) Manuscript writing: All authors; (VII) Final approval of \\ manuscript: All authors. \\ Correspondence to: Professor Xiong Liu; Xiaohong Peng. Department of Otolaryngology, Head and Neck Surgery, Nanfang Hospital, Southern \\ Medical University, 1838 Guangzhou Avenue North Road, Guangzhou 510515, China. Email: liux1218@126.com; 418897077@qq.com.
}

\begin{abstract}
Objective: To discuss the role and mechanism of vasculogenic mimicry (VM) and to provide reference for the further research of VM in head and neck tumors.

Background: Head and neck tumors are common in the clinic, and tumor metastasis is clinically difficult to treat. VM is another tumor blood supply mode that is different from angiogenesis and plays an important role in tumor growth, metastasis, and invasion. At present, studies on VM have mainly focused on breast cancer, melanoma, glioblastoma, and other cancers. With time, VM has become a hotspot in head and neck tumor research.

Methods: We searched published English literatures from 2015 to 2020 on PubMed. In this paper, we review the progress of VM in head and neck tumors from 7 different perspectives. VM has two distinct types, namely tubular type and patterned matrix type. VM is associated with high tumor grade, tumor progression, invasion, metastasis, and poor prognosis in patients with head and neck tumors. We discuss the recent studies on the effects of immune cells and Epstein-Barr virus on VM in head and neck tumors. Furthermore, we also summarize the molecular mechanism of VM formation in head and neck tumors. Finally, we discussed the possibility of VM-targeted therapy in the clinical treatment of head and neck tumors.

Conclusions: VM plays a critical role in tumor invasion, metastasis, and poor prognosis in patients with head and neck tumors. There is potential for VM as a potential new antitumor target. VM has become a hotspot in head and neck tumor research.
\end{abstract}

Keywords: Vasculogenic mimicry (VM); head and neck tumor; molecular mechanism

Submitted Jan 07, 2021. Accepted for publication May 10, 2021.

doi: $10.21037 /$ tcr-21-34

View this article at: https://dx.doi.org/10.21037/tcr-21-34

\section{Introduction}

Head and neck tumors are among the most common malignant tumors in the clinic, accounting for $2.8 \%$ of all malignant tumors, with an incidence of 15.22/100,000. Tumor metastasis is a difficult problem that has not yet been solved by the medical community and is also an important cause of death in patients with head and neck tumors. Although local control has improved due to advances in chemotherapy, radiotherapy, and surgical techniques, there has been little progress in head and neck cancer survival. Squamous cell carcinoma of the head and neck (HNSCC) is the most common histologic type, accounting for $90 \%$ of head and neck tumors, with a rate of synchronous and heterogeneous distant metastasis of 4-24\% (1).

Hematogenous metastasis is an important route of distant metastasis of advanced tumors, which depends on angiogenesis. The growth of both normal tissues and tumors

$\wedge$ ORCID: 0000-0002-5323-886X. 


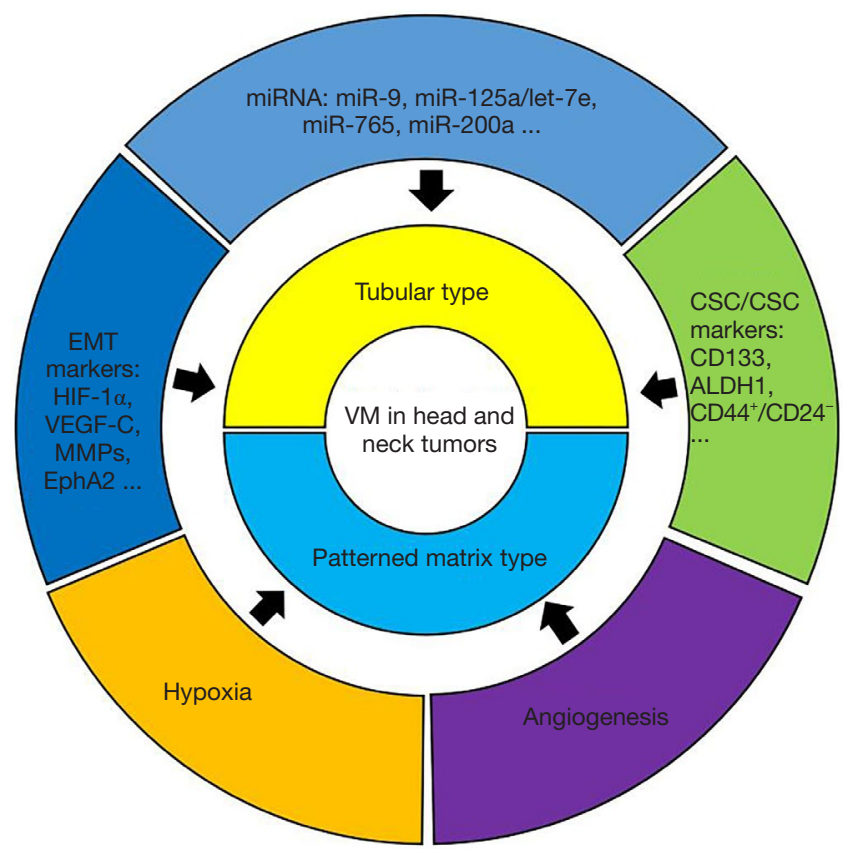

Figure 1 The types of vasculogenic mimicry (VM) and the factors that influence it. VM, vasculogenic mimicry; CSC, cancer stem cells; EMT, epithelial-mesenchymal transformation.

relies on blood vessels to supply oxygen and nutrients. Tumors larger than $2 \mathrm{~mm}$ in diameter need a blood supply or the cells will die from lack of oxygen. In a process known as angiogenesis, tumors secrete angiogenic factors that induce surrounding blood vessels to grow inside the tumor to provide nutrients (2). Based on these findings, scientists have developed antiangiogenic drugs to treat malignant tumors (3). As early as 1995, researchers began to investigate clinical possibility of anti-angiogenesis therapy (4). Antiangiogenic drugs are now being used in clinical trials aimed at fighting tumors. Lombardi et al. explored the effectiveness of anti-angiogenic drugs in patients with glioblastoma (5). In a review, Carla et al. summarized the role of antiangiogenic agents in clinical trials for head and neck cancer (6).

However, after a long period of clinical practice, doctors found that antiangiogenic drug therapy did not achieve the expected clinical efficacy in head and neck tumors and instead had the following results: (I) hemorrhage; (II) drug resistance; (III) inhibition but not elimination of the tumor; and (IV) unclear efficacy $(7,8)$. One theory is that antiangiogenic drugs are not effective because there may be other blood supply patterns for tumors, known as "vasculogenic mimicry (VM)".

In 1999, Maniotis et al. first proposed the concept of VM when they studied human melanoma $(2,9)$. Unlike angiogenesis, VM is not dependent on vascular endothelial cells (ECs) and can also transport oxygen and nutrients to tumor cells (10). In addition, VM formation in patients with malignant tumors is associated with tumor grade, invasion and metastasis, and poor prognosis. In recent years, VM has been reported in a variety of malignant tumors, such as melanoma, glioblastoma, osteosarcoma, liver cancer, breast cancer, lung cancer, gastric cancer, colorectal cancer, and prostate cancer $(2,11-17)$. The role of $\mathrm{VM}$ is very important for the progression of many tumors. Some studies have also shown that VM plays an important role in head and neck tumors, but relevant studies are lacking $(18,19)$. In this paper, we aimed to provide a summary of research on VM.

We present the following article in accordance with the Narrative Review reporting checklist (available at https:// dx.doi.org/10.21037/tcr-21-34).

\section{Methods}

We searched published English literatures from 2015 to 2020 on PubMed using the search terms: ("VM" OR "Vasculogenic mimicry") AND ("head and neck neoplasms" OR "head and neck cancer" OR "head and neck squamous cell cancer" OR "esophageal cancer" OR "esophageal squamous cell cancer" OR "oral cancer" OR "mouth neoplasms" OR "laryngeal neoplasms" OR "gingival neoplasms" OR "lip neoplasms" OR "palatal neoplasms" OR "tongue neoplasms" OR "pharyngeal neoplasms").

\section{Types of VM}

There are 2 types of VM (Figure 1): tubular type and patterned matrix type (2). The tubular type consists of tumor cells rather than ECs and is covered with secreted glycoproteins. The patterned matrix type has ducts that are made of extracellular matrix instead of cells. The patterned matrix type is a circular network formed by the stromal layer surrounding the tumor cell cluster. These layers are not evenly spaced, and as a result, fluid transport around the cell clusters is uneven. However, the matrix provides a larger diffusion surface area than the tubular structure, facilitating the delivery of nutrients and oxygen to tumor cells (20). 


\section{Factors influencing VM}

In order to help readers to better understand VM, the following list includes the currently known factors which affect VM (Figure 1).

\section{The role of cancer stem cells (CSCs) in VM}

In tumors, cells with the ability to self-renew and produce heterogeneous tumor cells are called CSCs, which play a decisive role in growth, metastasis, invasion, and prognosis of tumor therapy. The CSCs are characterized by their ability to self-renew and differentiate into different types of tumor cells. In recent years, researchers have found that CSCs play a key role in the formation of VM (21). Studies have shown that invasive melanoma cells that form VM appear to express genes associated with various cell phenotypes and stem cells, including epithelial, endothelial, muscle, neuronal, and other cell types. These CSC subgroups then differentiate/transdifferentiate and arrange the branched-lumen component (similar to the vascular network) to provide nutrition for the tumor. Finally, during angiogenesis or vascularization, these tubes extend and merge with blood vessels, allowing transport of blood cells. Multiple types of evidence have demonstrated that ECs can be produced from CSCs and transformed into ECs under conditions conducive to EC growth (22). Sun et al. showed that CSC markers in breast cancer such as CD133, $\mathrm{ALDH} 1$, and $\mathrm{CD} 44^{+} / \mathrm{CD} 24^{-}$were positively correlated with $\mathrm{VM}$ and the molecules involved in VM formation (23).

\section{The role of EMT in $V M$}

Epithelial-mesenchymal transformation (EMT) refers to the loss of cellular connections and polarity of epithelial cells and the acquisition of phenotypic characteristics of mesenchymal cells. Studies have shown that during VM formation, tumor cells involved in VM mimic the functions and phenotypes of ECs, which are a type of mesenchymal cell. Therefore, it is possible for tumor cells to transform from epithelial-type to mesenchymal-type ECs, namely, EMT. Many common regulatory factors have been reported between EMT and VM, such as hypoxia-inducible factor$1 \alpha$ (HIF-1 $\alpha)$, vascular endothelial growth factor (VEGF-C), and matrix metalloproteinases (MMPs) (24-26). Increasing evidence has shown that EMT is involved in the formation of VM $(27,28)$. Studies have shown that some biomolecules [such as erythromycin-producing hepatocyte receptor
(EphA2)] (29) can promote the formation of VM by regulating EMT, while inhibiting the expression of these biomolecules can hinder the formation of VM (30-32).

\section{The role of miRNA in VM}

In just over 2 decades since the discovery of the first microRNA (miRNA), the field of miRNA biology has expanded considerably. Insights into the roles of miRNAs in development and disease, particularly in cancer, have made miRNAs attractive tools and targets for novel therapeutic approaches. Functional studies have confirmed that miRNA dysregulation is causal in many cancers, with miRNAs acting as tumor suppressors or oncogenes (oncomiRs), and miRNA mimics and molecules targeting miRNAs (antimiRs) have shown promise in preclinical development (33). The miRNAs are a class of endogenous small RNAs approximately 20-24 nucleotides in length that have a variety of important regulatory roles in cells, such as early development, cell proliferation, apoptosis, fat metabolism, and cell differentiation. Studies have shown that miRNAs play a role (mostly inhibiting role) in the formation of VM (Table 1). For example, miR-9 can inhibit VM formation in gliomas. Experiments by Song et al. found that miR-9 inhibited the ability of tumor cells to form tubular structures in vitro, and this ability was restored with the addition of miR-9 inhibitors (34). Park et al.'s Studies showed that endothelial miR-125a/let7e suppresses interleukin-6 (IL-6)-induced adhesion of monocytes to ECs and induces paracrine inhibition of VM formation by cisplatin-treated EC conditioned media (35). Salinas-Vera et al. showed that miR-765 downregulates VEGF-A, AKT1, and SRC- $\alpha$ and directly targets VEGF-A to inhibit VM formation (36). Sun et al.'s results showed that miR-200a inhibits VM by negatively regulating EphA2 expression in ovarian cancer (37). Li et al. found that high expression of miR-124 inhibited VM formation in oral squamous cell carcinoma (OSCC) by reducing the production of p-STAT3 (38).

\section{Angiogenesis and VM}

Angiogenesis and VM are 2 similar but essentially different tumor blood supply patterns. There may be a potential link, but it is not clear yet. Hendrix et al. suggested that angiogenesis and VM are different manifestations of tumors at different stages of development. These researchers found that relatively inert uveal melanomas showed no signs 
Table 1 MiRNA in vasculogenic mimicry

\begin{tabular}{|c|c|c|c|c|c|}
\hline MiRNA & Cancer type & Target & Function characteristic & $\begin{array}{l}\text { Effect of VM } \\
\text { formation }\end{array}$ & Reference \\
\hline miR-9 & Gliomas & STMN1 & Targets the 3 '-UTR of STMN1 & Suppress & (34) \\
\hline miR-765 & Ovarian cancer & VEGFR & $\begin{array}{c}\text { MiR-765 downregulates VEGFA, AKT1 and SRC- } \alpha \text { and directly } \\
\text { target VEGFA }\end{array}$ & Suppress & (36) \\
\hline miR-200a & Ovarian cancer & EphA2 & Negatively regulating EphA2 & Suppress & (37) \\
\hline miR-124 & $\begin{array}{l}\text { Oral squamous cell } \\
\text { carcinoma }\end{array}$ & p-STAT3 & Reducing p-STAT3 production & Suppress & (38) \\
\hline
\end{tabular}

ECs, endothelial cells; VM, vasculogenic mimicry.

of VM, but invasive tumors developed microcirculation composed of cell-free vascular channels produced by the tumor cells themselves, which generally prevented necrosis, even in relatively large tumors. The channels produced in this form can be connected to pre-existing blood vessels associated with the tumor (10).

\section{Hypoxia and VM}

Due to the unrestrained growth of tumors and the special metabolic characteristics of tumor cells, tumor tissues consume a large amount of nutrients and oxygen, which allows tumor growth in the hypoxic microenvironment. Studies have shown that such an anoxic microenvironment can promote the formation of VM. The HIFs and hypoxiaresponsive elements (HREs) play important roles in the formation of tumor VM. Some genes involved in VM formation, including Twist, E-cadherin, Nodal, EphA2, and VEGF-A, have been found to be regulated by HIFs or HREs (2).

\section{The influence of VM on the prognosis of head and neck tumors}

Cancer metastasis is the leading cause of cancer-related morbidity and mortality, accounting for approximately $90 \%$ of cancer-specific deaths. Although several approaches have been used in the treatment of patients with head and neck tumors, overall survival (OS) is low, especially when lymph node metastasis (LNM) or recurrence occurs. The presence of VM has an important impact on the growth and metastasis of tumors, so understanding the influence of VM on the prognosis of tumors is important for clinicians to develop more effective treatment programs.

Hujanen et al. used a meta-analysis to investigate the effect of VM on the prognosis of HNSCC (39). A total of 7 studies were selected from 117 studies, and then the data of these 7 studies were analyzed. First, VM was believed to be significantly positively correlated with tumor size, grade, LNM, and TNM (tumor, lymph node, and metastasis) stage. The presence of VM was more common in patients with local LNM or an advanced stage of pathology. In addition, VM positivity (as opposed to VM negativity) was associated with poorer survival. People with VM-positive status were more likely to die early than those with VMnegative status (hazard ratio $=0.47$ ). Chen $e t$ al. found that among patients with orbital tumors, $\mathrm{VM}(+)$ patients had a significantly reduced survival rate, significantly increased risk of death, and increased EphA2 and MMP-2 expression levels (EphA2 and MMP-2 can promote the formation of tumor VM) (40). Wang et al. found that in OSCC, the VM positive rate was positively correlated with tumor grade and primary tumor (PT), LNM, and TNM stage and negatively correlated with the patient's OS time (41).

\section{Effects of immune cells on VM in head and neck tumors}

During carcinogenesis, advanced tumors are surrounded by stromal cells and immune cells that support tumor development. Immune cells participate in the formation of VM by releasing angiogenic factors and promoting early tumor vascularization. Among the major immune cells involved in these mechanisms, tumor-associated macrophages (TAMs) secrete MMPs, which promote remodeling of the extracellular matrix and facilitate the 
Table 2 Molecular mechanism of vasculogenic mimicry formation in head and neck tumors

\begin{tabular}{|c|c|c|c|c|c|}
\hline Molecular & Cancer type & Target & Function & $\begin{array}{l}\text { Effect of VM } \\
\text { formation }\end{array}$ & Reference \\
\hline HIF- $1 \alpha$ & $\begin{array}{c}\text { Nasopharyngeal } \\
\text { carcinoma }\end{array}$ & PI3K-AKT-mTOR & $\begin{array}{l}\text { The ability of cells to metastasize, invade, and form } \\
\text { tubular structures was reduced in a dose-dependent } \\
\text { manner after adding the HIF- } 1 \alpha \text { inhibitor }\end{array}$ & Promote & (18) \\
\hline EphA2 & $\begin{array}{l}\text { Squamous cell } \\
\text { carcinoma }\end{array}$ & $\begin{array}{l}\text { EMT-related } \\
\text { molecules }\end{array}$ & $\begin{array}{l}\text { The expression of Twist and Vimentin was found to rise, } \\
\text { while the expression of E-cadherin and claudin- } 4 \text { was } \\
\text { downregulated }\end{array}$ & Promote & (29) \\
\hline JAK2/STAT3 & $\begin{array}{l}\text { Laryngeal } \\
\text { cancer }\end{array}$ & MMP2, VEGF, etc. & $\begin{array}{l}\text { Regulate the expression of MMP-2, VEGF and other } \\
\text { genes }\end{array}$ & Promote & (44) \\
\hline
\end{tabular}

VM, vasculogenic mimicry; EMT, epithelial-mesenchymal transition.

movement and invasion of tumor cells. In addition, TAMs release a range of cytokines, including transforming growth factor-beta (TGF- $\beta$ ), tumor necrosis factor-alpha (TNF- $\alpha$ ), interleukin-1 beta (IL-1ß), IL-6, and interleukin-8 (IL-8), which contribute to EMT activation (42). Other immune cells involved in the activity of these cells include tumorassociated neutrophils (TANs) and marrow-derived suppressor cells (MDSCs). These immune cells and their secreted molecules then activate the PI $3 \mathrm{~K}$ and NF- $\mathrm{NB}$ signaling pathways to promote EMT and VM (2).

Shi et al.'s study showed that in patients with sinus cancer, $\mathrm{VM}(+)$ patients had more TAM infiltration in their tumor tissues than VM(-) patients (42). Pang et al. found that after 48 hours of in vitro 3D culture of tumor-associated MDSCs, OSCC cells formed a typical vascular-like structure. In addition, compared with MDSCs from healthy donors, tumor-related MDSCs increased the expression of vascular endothelial (VE)-cadherin in OSCC cells, which is a characteristic of ECs. This finding suggests that MDSCs derived from OSCC may promote the formation of $\mathrm{VM}$ in OSCC cells by inducing EMT (43).

\section{Effects of EB virus on VM in head and neck tumors}

At present, there are approximately 30 kinds of known oncogenic viruses, among which the Epstein-Barr virus (EBV) is the most common in the field of head and neck tumors. The EBV is a pneumotropic virus in the herpesvirus family. It has a DNA genome. The EBV exclusively infects humans and some primate $\mathrm{B}$ cells in vitro and in vivo. Humans are hosts of EBV infection, which is mainly transmitted through saliva. Nasopharyngeal carcinoma (NPC) is a common epithelial malignancy closely related to
EBV. NPC accounts for $70 \%$ of EBV-associated epithelial malignancies. It mostly occurs in middle-aged and elderly people over the age of 40, and NPC has a high incidence in south China (Guangdong, Guangxi, Fujian, etc.) and Southeast Asia.

However, the exact role of EBV in epithelial cancer remains unclear. Xiang et al. demonstrated the function of EBV in VM (18). First, when we examined the morphology of individual nasopharyngeal cancer cells, $\mathrm{EBV}(+)$ cells were elongated, while EBV(-) cells were shaped like pebbles. When the cells were then planted on 3D medium, EBV (+) cells formed a circular network, and EBV(-) cells were distributed evenly in the medium. To further clarify the structure of the tubular network, we examined the medium by scanning electron microscope (SEM). This method confirmed that the structure formed by $\mathrm{EBV}(+)$ NPC cells was indeed tubular and lined with tumor cells. These nasopharyngeal cancer cells were then subcutaneously injected into mice, and tissue samples were taken 20 days later. Staining with CD31 and Periodic Acid-Schiff (PAS) revealed that $\mathrm{EBV}(+)$ tumors had a larger volume and more PAS(+)/CD31(-) tubular structures than EBV(-) tumors. The relationship among EBV infection, VM formation, and AKT/HIF-1 $\alpha$ pathway inhibitory activation was also demonstrated. This finding will be mentioned below.

\section{Molecular mechanism of VM formation in head and neck tumors}

At present, the molecules involved in VM formation in head and neck tumors that have been studied include EphA2, JAK2, STAT, miR-124, and so on (Table 2).

The molecule EphA2 is a transmembrane tyrosine kinase receptor, a member of the tyrosine kinase receptor 
(RTK) superfamily. Wang et al. demonstrated that EphA2 participates in the formation of VM by regulating EMT in HNSCC (29). First, they found that downregulation of EphA2 expression can destroy the formation of channellike structures, while upregulation of EphA2 expression can restore their function. Moreover, the expression of EMTrelated molecules was detected; the expression of Twist and Vimentin was found to rise, while that of E-cadherin and claudin-4 was downregulated, indicating that EphA2 could regulate the formation of VM and that EMT was involved.

The JAK2/STAT-3 pathway is an important pathway involved in VM formation in head and neck tumors, and JAK2 is a member of the nonreceptor tyrosine kinase (Janus kinase) family. Activation of JAK2 by external stimulus signals resulted in the recruitment and phosphorylation of the STAT protein to $\mathrm{p}-\mathrm{STAT}$. The p-STAT protein can enter the nucleus and regulate the expression of MMP-2, VEGF, and other genes. It has been shown that STAT-3 is an important mediator of VM. Hu et al. demonstrated that inhibiting the JAK2/STAT-3 pathway effectively prevented VM formation in laryngeal cancer (44). When the JAK2 inhibitor AG490 was added to the culture medium, the formation of VM of tumor cells was significantly decreased. Furthermore, the amount of p-STAT was significantly reduced, as shown by western blot analysis, while the amount of total STAT-3 protein was unchanged.

The miRNAs play a key role in the formation of VM. Li et al. found that high expression of miR-124 inhibited VM formation in OSCC by reducing the production of p-STAT3 (38). The oral cancer cell line HN6-miR-124 overexpressing miR-124 was established by plasmid vector transfection. Compared with that of the control group (HN6-miR-Ctrl), the production of p-STAT3 in HN6miR-124 cells was significantly reduced, as was the ability of oral cancer cells to metastasize, invade, and form tubular structures.

In addition to these molecules, other molecules play an important role in the formation of VM in head and neck tumors. Xiang et al. suggested that HIF-1 $\alpha$ involvement is required in VM formation induced by EBV. The EBV increases HIF- $1 \alpha$ synthesis through the PI3K-AKT-mTOR pathway (18). They used 2 HIF- $1 \alpha$ inhibitors to treat NPC cells, and the ability of cells to metastasize, invade, and form tubular structures was reduced in a dose-dependent manner. In addition, HIF- $1 \alpha$-associated genes can be knocked out with specific small interfering RNAs (siRNAs), and the same results were obtained.

In summary, the molecular mechanism of VM formation in head and neck tumors is very complex and has not been fully elucidated. These processes require further exploration and study.

\section{VM targeted therapy is a new idea for the clinical treatment of head and neck tumors}

There are currently a variety of treatments for head and neck tumors that have been shown to have good results; however, some cases still show poor treatment results. The presence of VM is closely related to tumor growth, metastasis and invasion, and its discovery has provided us with new treatment strategies.

Li et al. demonstrated that niclosamide could inhibit VM formation by upregulating miR-124 expression and simultaneously reduce the ability of oral cancer cells to invade and metastasize (38). Liu et al. investigated the effect of melatonin inhibition of reactive oxygen species on VM formation in oral cancer cells. Melatonin inhibits VM in oral cancer cells by blocking the reactive oxygen-dependent ERK/Akt signaling pathway (45). Hu et al. showed that curcumin inhibits the aggressiveness and VM of laryngeal squamous cell carcinoma by inhibiting the JAK2/STAT-3 signaling pathway (44). Xiang et al. showed that traditional antiangiogenic drugs could suppress NPC cells, forming a mesh structure in $3 \mathrm{D}$ medium, and the combination of antiangiogenic drugs and drugs targeting VM can effectively inhibit NPC cell invasion, microvascular formation, and $\mathrm{VM}$; this effect is preferable to that of single antiangiogenic drugs or drugs targeting VM (18).

\section{Summary}

In this paper, we reviewed the research progress of VM formation in head and neck tumors in recent years and various mechanisms regulating VM formation in tumors. At present, studies on VM in head and neck tumors have not matched the quantity and quality of those investigating VM in other cancers, such as breast cancer, non-small cell lung cancer, and glioblastoma. Compared to head and neck cancers, there appears to be greater interest in investigating lung cancer, liver cancer, breast cancer, and other cancers that affect more people and are more widely distributed. The lack of interest in the study of head and neck tumors is an important reason for the lack of high-quality articles to date.

However, increasing evidence has shown that VM plays a critical role in tumor invasion, metastasis, and 
poor prognosis in patients with head and neck tumors. There is potential for $\mathrm{VM}$ as a new antitumor target. However, the mechanism of VM facilitation is not yet fully understood. It has been concluded that antiangiogenic therapy is still not ideal due to the formation of VM, and that some VM-targeted drugs can effectively inhibit tumor progression. Therefore, the clinical application of novel molecular compounds targeting tumor VM needs to be further explored. The combination of VM-targeted drugs and antiangiogenic therapy may provide a promising new direction in cancer therapy.

\section{Acknowledgments}

We thank our anonymous reviewers for their valuable comments, which have led to many improvements to this article.

Funding: This study was supported by grants from the National Natural Science Foundation of China (81702696), Natural Science Foundation of Guangdong Province of China (2017A030310040 and 2020A1515010176), and Medical Scientific Research Foundation of Guangdong Province of China (A2020078).

\section{Footnote}

Reporting Checklist: The authors have completed the Narrative Review reporting checklist. Available at https:// dx.doi.org/10.21037/tcr-21-34

Conflicts of Interest: All authors have completed the ICMJE uniform disclosure form (available at https://dx.doi. org/10.21037/tcr-21-34). The authors have no conflicts of interest to declare.

Ethical Statement: The authors are accountable for all aspects of the work in ensuring that questions related to the accuracy or integrity of any part of the work are appropriately investigated and resolved.

Open Access Statement: This is an Open Access article distributed in accordance with the Creative Commons Attribution-NonCommercial-NoDerivs 4.0 International License (CC BY-NC-ND 4.0), which permits the noncommercial replication and distribution of the article with the strict proviso that no changes or edits are made and the original work is properly cited (including links to both the formal publication through the relevant DOI and the license).
See: https://creativecommons.org/licenses/by-nc-nd/4.0/.

\section{References}

1. Tang E, Lahmi L, Meillan N, et al. Treatment Strategy for Distant Synchronous Metastatic Head and Neck Squamous Cell Carcinoma. Curr Oncol Rep 2019;21:102.

2. Luo Q, Wang J, Zhao W, et al. Vasculogenic mimicry in carcinogenesis and clinical applications. J Hematol Oncol 2020;13:19.

3. Lee AW, Ma BB, Ng WT, et al. Management of Nasopharyngeal Carcinoma: Current Practice and Future Perspective. J Clin Oncol 2015;33:3356-64.

4. Gasparini G, Harris AL. Clinical Importance of the Determination of Tumor Angiogenesis in Breast Carcinoma: Much More than a New Prognostic Tool. J Clin Oncol 1995;13:765-82.

5. Lombardi G, Pambuku A, Bellu L, et al. Effectiveness of Antiangiogenic Drugs in Glioblastoma Patients: A Systematic Review and Meta-Analysis of Randomized Clinical Trials. Crit Rev Oncol Hematol 2017;111:94-102.

6. Carla C, Daris F, Cecilia B, et al. Angiogenesis in Head and Neck Cancer: A Review of the Literature. J Oncol 2012;2012:358472.

7. Lugano R, Ramachandran M, Dimberg A. Tumor Angiogenesis: Causes, Consequences, Challenges and Opportunities. Cell Mol Life Sci 2020;77:1745-70.

8. Kuczynski EA, Reynolds AR. Vessel Co-Option and Resistance to Anti-Angiogenic Therapy. Angiogenesis 2020;23:55-74.

9. Maniotis AJ, Folberg R, Hess A, et al. Vascular channel formation by human melanoma cells in vivo and in vitro: vasculogenic mimicry. Am J Pathol 1999;155:739-52.

10. Folberg R, Hendrix MJC, Maniotis AJ. Vasculogenic Mimicry and Tumor Angiogenesis. Am J Pathol 2000;156:361-81.

11. Seftor EA, Meltzer PS, Schatteman GC, et al. Expression of multiple molecular phenotypes by aggressive melanoma tumor cells: role in vasculogenic mimicry. Crit Rev Oncol Hematol 2002;44:17-27.

12. Plate KH, Scholz A, Dumont DJ. Tumor Angiogenesis and Anti-Angiogenic Therapy in Malignant Gliomas Revisited. Acta Neuropathologica 2012;124:763-75.

13. Ren K, Ni Y, Li X, et al. Expression Profiling of Long Noncoding RNAs Associated with Vasculogenic Mimicry in Osteosarcoma. J Cell Biochem 2019;120:12473-88.

14. Xiao T, Zhong W, Zhao J, et al. Polyphyllin I Suppresses the Formation of Vasculogenic Mimicry Via Twist1/VE- 
cadherin Pathway. Cell Death Dis 2018;9:906.

15. Andonegui-Elguera MA, Alfaro-Mora Y, CáceresGutiérrez R, et al. An Overview of Vasculogenic Mimicry in Breast Cancer. Front Oncol 2020;10:220.

16. Williamson SC, Metcalf RL, Trapani F, et al. Vasculogenic mimicry in small cell lung cancer. Nat Commun 2016;7:13322.

17. Li Y, Wu Z, Yuan J, et al. Long Non-Coding RNA MALAT1 Promotes Gastric Cancer Tumorigenicity and Metastasis by Regulating Vasculogenic Mimicry and Angiogenesis. Cancer Lett 2017;395:31-44.

18. Xiang T, Lin Y, Ma W, et al. Vasculogenic Mimicry Formation in EBV-associated Epithelial Malignancies. Nat Commun 2018;9:5009-15.

19. Xu S, Bai J, Zhuan Z, et al. EBV-LMP1 is Involved in Vasculogenic Mimicry Formation Via VEGFA/VEGFR1 Signaling in Nasopharyngeal Carcinoma. Oncol Rep 2018;40:377-84.

20. Ayala-Domínguez L, Olmedo-Nieva L, Muñoz-Bello JO, et al. Mechanisms of Vasculogenic Mimicry in Ovarian Cancer. Front Oncol 2019;9:998.

21. Yao XH, Ping YF, Bian XW. Contribution of cancer stem cells to tumor vasculogenic mimicry. Protein Cell 2011;2:266-72.

22. Krishna Priya S, Nagare RP, Sneha VS, et al. Tumour angiogenesis-Origin of blood vessels. Int J Cancer 2016;139:729-35.

23. Sun H, Yao N, Cheng S, et al. Cancer Stem-Like Cells Directly Participate in Vasculogenic Mimicry Channels in Triple-Negative Breast Cancer. Cancer Biol Med 2019;16:299-311.

24. Wechman SL, Emdad L, Sarkar D, et al. Vascular Mimicry: Triggers, Molecular Interactions and in Vivo Models. Adv Cancer Res 2020;148:27-67.

25. Wu ZZ, Chen LS, Zhou R, et al. Metastasis-Associated in Colon Cancer-1 in Gastric Cancer: Beyond Metastasis. World J Gastroenterol 2016;22:6629-37.

26. Zhang S, Zhang D, Sun B. Vasculogenic Mimicry: Current Status and Future Prospects. Cancer Lett 2007;254:157-64.

27. Orecchia P, Balza E, Pietra G, et al. L19-IL2 Immunocytokine in Combination with the AntiSyndecan-1 46F2SIP Antibody Format: A New Targeted Treatment Approach in an Ovarian Carcinoma Model. Cancers (Basel) 2019;11:1232.

28. Zhao J, Wu J, Qin Y, et al. LncRNA PVT1 induces aggressive vasculogenic mimicry formation through activating the STAT3/Slug axis and epithelial-to- mesenchymal transition in gastric cancer. Cell Oncol (Dordr) 2020;43:863-76.

29. Wang W, Lin P, Sun B, et al. Epithelial-mesenchymal transition regulated by EphA2 contributes to vasculogenic mimicry formation of head and neck squamous cell carcinoma. Biomed Res Int 2014;2014:803914.

30. Wan HY, Li QQ, Zhang Y, et al. MiR-124 represses vasculogenic mimicry and cell motility by targeting amotL1 in cervical cancer cells. Cancer Lett 2014;355:14858. Erratum in: Cancer Lett. 2021 May 1;505:91-93. doi: 10.1016/j.canlet.2021.02.014. Epub 2021 Mar 4.

31. Wang M, Zhao X, Zhu D, et al. HIF-1 $\alpha$ promoted vasculogenic mimicry formation in hepatocellular carcinoma through LOXL2 up-regulation in hypoxic tumor microenvironment. J Exp Clin Cancer Res 2017;36:60.

32. Hou F, Li W, Shi Q, et al. Yi Ai Fang, a traditional Chinese herbal formula, impacts the vasculogenic mimicry formation of human colorectal cancer through HIF-1 and epithelial mesenchymal transition. BMC Complement Altern Med 2016;16:428.

33. Rupaimoole R, Slack FJ. MicroRNA Therapeutics: Towards a New Era for the Management of Cancer and Other Diseases. Nat Rev Drug Discov 2017;16:203-22.

34. Song Y, Mu L, Han X, et al. MicroRNA-9 inhibits vasculogenic mimicry of glioma cell lines by suppressing Stathmin expression. J Neurooncol 2013;115:381-90.

35. Park Y, Kim J. Regulation of IL-6 Signaling by miR-125a and Let-7e in Endothelial Cells Controls Vasculogenic Mimicry Formation of Breast Cancer Cells. BMB Rep 2019;52:214-9.

36. Salinas-Vera YM, Gallardo-Rincon D, Garcia-Vazquez $\mathrm{R}$, et al. HypoxamiRs Profiling Identify miR-765 as a Regulator of the Early Stages of Vasculogenic Mimicry in SKOV3 Ovarian Cancer Cells. Front Oncol 2019;9:381.

37. Sun Q, Zou X, Zhang T, et al. The Role of miR-200a in Vasculogenic Mimicry and its Clinical Significance in Ovarian Cancer. Gynecol Oncol 2014;132:730-8.

38. Li X, Yang Z, Han Z, et al. Niclosamide Acts as a New Inhibitor of Vasculogenic Mimicry in Oral Cancer through Upregulation of miR-124 and Downregulation of STAT3. Oncol Rep 2018;39:827-33.

39. Hujanen R, Almahmoudi R, Karinen S, et al. Vasculogenic Mimicry: A Promising Prognosticator in Head and Neck Squamous Cell Carcinoma and Esophageal Cancer? A Systematic Review and Meta-Analysis. Cells 2020;9:507.

40. Chen L, He Y, Sun S, et al. Vasculogenic Mimicry is a Major Feature and Novel Predictor of Poor Prognosis in 
Patients with Orbital Rhabdomyosarcoma. Oncol Lett 2015;10:1635-41.

41. Wang Y, Wang X, Zhang Y, et al. Vasculogenic Mimicry and Expression of ALDH1, Beclin1, and P16 Correlate with Metastasis and Prognosis in Oral Squamous Cell Carcinoma. Int J Clin Exp Pathol 2018;11:1599-609.

42. Shi L, Lei D, Ma C, et al. Clinicopathological implications of tumour-associated macrophages and vascularization in sinonasal melanoma. J Int Med Res 2010;38:1276-86.

43. Pang X, Fan HY, Tang YL, et al. Myeloid derived suppressor cells contribute to the malignant progression

Cite this article as: Yue Y, Lou Y, Liu X, Peng X. Vasculogenic mimicry in head and neck tumors: a narrative review. Transl Cancer Res 2021;10(6):3044-3052. doi: 10.21037/tcr-21-34 of oral squamous cell carcinoma. PLoS One 2020;15:e0229089.

44. Hu A, Huang JJ, Jin XJ, et al. Curcumin suppresses invasiveness and vasculogenic mimicry of squamous cell carcinoma of the larynx through the inhibition of JAK-2/STAT-3 signaling pathway. Am J Cancer Res 2014;5:278-88.

45. Liu R, Wang HL, Deng MJ, et al. Melatonin Inhibits Reactive Oxygen Species-Driven Proliferation, EpithelialMesenchymal Transition, and Vasculogenic Mimicry in Oral Cancer. Oxid Med Cell Longev 2018;2018:3510970. 